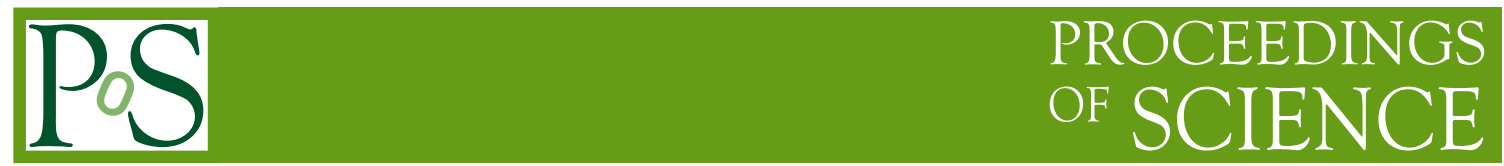

\title{
Structure changes of hadrons in nuclear matter
}

\author{
Ulugbek Yakhshiev* \\ Department of Physics, Inha University, Incheon 402-751, Republic of Korea \\ E-mail: yakhshiev@inha.ac.kr
}

We discuss the properties of in-medium nucleons in the framework of the chiral solitonic approach. The approach is based on the in-medium modified version of the Skyrme model which takes into account "outer shell" as well as "inner core" modifications of the in-medium nucleons. The modifications of the in-medium properties of nucleons are performed in close connection with nuclear matter properties.

XV International Conference on Hadron Spectroscopy-Hadron 2013

4-8 November 2013

Nara, Japan

${ }^{*}$ Speaker. 


\section{Introduction}

The studies of hadron properties at finite density and temperature are important for understanding the properties of strongly interacting matter which may be formed during the relativistic heavy-ion collisions. First observations of the structure changes of the in-medium nucleons by European Muon Collaboration [1] initiated further experimental measurements and different theoretical approaches. Most of the theoretical approaches are based on in-medium nucleons perceiving their individuality (for further references, see the recent work [2] ). In this context, the possible in-medium modifications of the low energy constants of the truncated effective chiral Lagrangian could reproduce nuclear matter properties [2]. The results are found to be in good agreement with the results of successful phenomenological approaches [3, 4].

The basic idea behind of the approach developed in Ref. [2] was the structure changes of the in-medium nucleons due to the influence of surrounding nuclear medium. In achieving nuclear matter properties, it was taken into account the possible "outer shell" changes of the in-medium nucleons at low densities with further inclusion of "internal core" changes at densities close to the normal nuclear matter density $\rho_{0} \sim 0.16 \mathrm{fm}^{-3}$, and at supra-normal nuclear matter densities. Extensions of the model including the explicit vector mesonic degrees of freedom also gave the satisfactory results in reproducing nuclear matter properties and predicted the possible changes in the in-medium vector meson properties [5].

In this context, it will be interesting to revise the in-medium properties of the single nucleon in a self-consistent way while nuclear matter properties already satisfactorily reproduced [2]. Therefore, in the present work we will concentrate on the in-medium nucleon properties.

\section{Lagrangian of the model}

Our starting point is the in-medium modified Skyrme-model Lagrangian described in Ref. [2] where we assumed that the pionic sector has the mass degeneracy, $m_{\pi^{0}}=m_{\pi^{ \pm}}$. Due to this approximation our calculations become very simple and transparent. The resulting Lagrangian has form $^{1}$

$$
\begin{aligned}
\mathscr{L}^{*} & =\mathscr{L}_{2}^{*}+\mathscr{L}_{4}^{*}+\mathscr{L}_{m}^{*}+\mathscr{L}_{e}^{*}, \\
\mathscr{L}_{2}^{*} & =\frac{F_{\pi}^{2}}{16} \alpha_{\tau} \operatorname{Tr}\left(\partial_{0} U \partial_{0} U^{\dagger}\right)-\frac{F_{\pi}^{2}}{16} \alpha_{s} \operatorname{Tr}\left(\partial_{i} U \partial_{i} U^{\dagger}\right), \\
\mathscr{L}_{4}^{*} & =-\frac{1}{16 e^{2} \zeta_{\tau}} \operatorname{Tr}\left[U^{\dagger} \partial_{0} U, U^{\dagger} \partial_{i} U\right]^{2}+\frac{1}{32 e^{2} \zeta_{s}} \operatorname{Tr}\left[U^{\dagger} \partial_{i} U, U^{\dagger} \partial_{j} U\right]^{2}, \\
\mathscr{L}_{m}^{*} & =-\frac{F_{\pi}^{2} m_{\pi}^{2}}{16} \alpha_{m} \operatorname{Tr}\left(2-U-U^{+}\right), \\
\mathscr{L}_{e}^{*} & =-\frac{F_{\pi}^{2}}{16} m_{\pi} \alpha_{e} \varepsilon_{a b 3} \operatorname{Tr}\left(\tau_{a} U\right) \operatorname{Tr}\left(\tau_{b} \partial_{0} U^{\dagger}\right) .
\end{aligned}
$$

Here the chiral $\mathrm{SU}(2)$ matrix $U=\exp \left(2 i \tau_{a} \pi_{a} / F_{\pi}\right)$ is defined in terms of the Cartesian isospincomponents of the pion field $\pi_{a}(a=1,2,3)$. The density functionals entering into the Lagrangian $\left(\alpha_{\tau}, \alpha_{s}, \zeta_{\tau}\right.$, etc.) represent the influence of surrounding environment to the single soliton properties. Their relations to the low energy pion-nucleon scattering data and to nuclear matter properties, the

\footnotetext{
${ }^{1}$ Hereafter, "asterisk" in an expression means the explicit medium modification.
} 
minimization procedure, the quantization of solution and other details can be found in Ref. [2]. Here we present only some final formulas and concentrate our attention to the outcome results related to the in-medium nucleon properties.

In the single skyrmonic sector, the input parameters of the model have the following values: $F_{\pi}=108.783 \mathrm{MeV}, e=4.854$ and $m_{\pi}=134.976 \mathrm{MeV}$. They correctly reproduce the experimental values of the nucleon mass $m_{N}=938 \mathrm{MeV}$ and $\Delta$-isobara mass $m_{\Delta}=1232 \mathrm{MeV}$ in free space, i.e. if $\alpha_{\tau}=\alpha_{s}=\alpha_{m}=\zeta_{\tau}=\zeta_{s}=1$ and $\alpha_{e}=0$.

While our aim is the analysis of in-medium properties of the nucleons in homogeneous and infinite nuclear matter, all medium functionals become the functions of density or simple the free parameters. In this approximation one can use spherically symmetric hedgehog ansatz $U=$ $\exp \{i \vec{\tau} \vec{n} F(r)\}$ and the mass of classical soliton at a given density takes form

$$
M^{*}=\sqrt{\frac{\alpha_{s}}{\zeta_{s}}}\left(\frac{\pi F_{\pi}}{e}\right) \int_{0}^{\infty} d x x^{2}\left\{2 \beta^{2}(1-\cos F)+\frac{F_{x}^{2}}{2}+\frac{\sin ^{2} F}{x^{2}}+\frac{2 \sin ^{2} F}{x^{2}}\left[2 F_{x}^{2}+\frac{\sin ^{2} F}{x^{2}}\right]\right\} .
$$

Here we introduced the notation $F_{x}=\partial F / \partial x$ and the scaled variable $x=e F_{\pi} r\left(\alpha_{s} \zeta_{s}\right)^{1 / 2}$. The parameter $\beta$ contains the density functions and has form

$$
\beta^{2}=\left(\frac{m_{\pi}^{2}}{e^{2} F_{\pi}^{2}}\right) \frac{\alpha_{m}}{\left(\alpha_{s}\right)^{2} \zeta_{s}} \equiv \beta_{0}^{2} \frac{\alpha_{m}}{\left(\alpha_{s}\right)^{2} \zeta_{s}}
$$

The minimization of the mass functional (2.2) with the corresponding boundary conditions (e.g., baryon number equal to one $B=1$ condition) gives the in-medium-solitonic solutions.

\section{Quantization, in-medium nucleons and nuclear matter}

Considering the time dependent isospin rotations

$$
U \rightarrow U(t)=A(t) U A^{\dagger}(t), \quad A=\exp \left\{\frac{i \vec{\tau} \vec{\omega}}{2}\right\}
$$

and defining the canonical conjugate variables $T_{i}=\partial L^{*} / \partial \omega_{i}$ one obtains from the time-dependent Lagrangian the following Hamiltonian

$$
\hat{H}=M^{*}+\frac{\Lambda_{e}^{* 2}}{2 \Lambda^{*}}+\frac{\hat{\vec{T}}^{2}}{2 \Lambda^{*}}-\frac{\Lambda_{e}^{*}}{\Lambda^{*}} \hat{T}_{3},
$$

where the in-medium moments of inertia are given by expressions

$$
\Lambda^{*}=\left(\alpha_{s} \zeta_{s}\right)^{-3 / 2}\left(\alpha_{\tau} \Lambda_{2}+\zeta_{\tau}^{-1} \alpha_{s} \zeta_{s} \Lambda_{4}\right), \quad \Lambda_{e}^{*}=\left(\alpha_{s} \zeta_{s}\right)^{-3 / 2} m_{\pi} \alpha_{e} \Lambda_{2}
$$

Here $\Lambda_{2}$ and $\Lambda_{4}$ are schematically keeping their free space forms

$$
\begin{aligned}
& \Lambda_{2}=\frac{2 \pi}{3 e^{3} F_{\pi}} \int_{0}^{\infty} x^{2} \sin ^{2} F d x, \\
& \Lambda_{4}=\frac{8 \pi}{3 e^{3} F_{\pi}} \int_{0}^{\infty}\left(F_{x}^{2}+\frac{\sin ^{2} F}{x^{2}}\right) x^{2} \sin ^{2} F d x .
\end{aligned}
$$


The sandwiching of the Hamiltonian between the appropriate baryon states $\left|T, T_{3} ; J, J_{3}\right\rangle$ determines the in-medium masses of baryons. For example, an in-medium nucleon mass has form

$$
\begin{aligned}
m_{N}^{*}(\rho, \delta \rho) & =m_{N, s}^{*}(\rho, \delta \rho)-2 m_{N, v}^{*}(\rho, \delta \rho) T_{3}, \\
m_{N, s}^{*}(\rho, \delta \rho) & \equiv M^{*}(\rho)+\frac{\Lambda_{e}^{* 2}(\delta \rho)}{2 \Lambda^{*}(\rho)}+\frac{3}{8 \Lambda^{*}(\rho)}, \quad m_{N, v}^{*}(\rho, \delta \rho) \equiv \frac{\Lambda_{e}^{*}(\delta \rho)}{2 \Lambda^{*}(\rho)},
\end{aligned}
$$

where $T_{3}$ is the third component of isospin, $\rho=\rho_{\mathrm{n}}+\rho_{\mathrm{p}}$ and $\delta \rho=\rho_{\mathrm{n}}-\rho_{\mathrm{p}}$ are the isoscalar and the isovector density distributions of the surrounding nuclear medium, respectively. One can see that due to the isospin asymmetry of environment the in-medium masses of neutrons and protons do not degenerate anymore. Note, that in free space the nucleons degenerate in mass.

Further, using one simplifying condition $\alpha_{\tau}=\zeta_{\tau}^{-1} \alpha_{s} \zeta_{s}$ and parametrizing the four combinations of medium functions

$$
\begin{aligned}
& f_{1}=\sqrt{\frac{\alpha_{s}}{\zeta_{s}}}=1+C_{1} \lambda, \quad f_{2}=\sqrt{\frac{\alpha_{m}}{\left(\alpha_{s}\right)^{2} \zeta_{s}}}=1+C_{2} \lambda, \quad f_{3}=\frac{\alpha_{\tau}}{\left(\alpha_{s} \zeta_{s}\right)^{3 / 2}}=1+C_{3} \lambda, \\
& \frac{\alpha_{e}}{\alpha_{\tau}} \equiv f_{4} \delta=\frac{C_{4} \lambda}{1+C_{5} \lambda} \delta
\end{aligned}
$$

one can easily reproduce the symmetric and asymmetric matter properties $[2,6]$. Here $C_{i}, i=\overline{1,5}$ are the variational parameters, $\lambda=\rho / \rho_{0}$ is the density parameter and $\delta=\delta \rho / \rho$ is the asymmetry parameter. More details can be found in Refs. [2, 6].

\section{Results and discussions}

Defining the binding energy per nucleon in symmetric nuclear matter as

$$
\varepsilon_{V}(\lambda)=m_{N}^{*}(\lambda, 0)-m_{N}
$$

one can fit the values of parameters $C_{1}, C_{2}$ and $C_{3}$ from the depth of binding energy $\varepsilon\left(\rho_{0}\right)=$ $-16 \mathrm{MeV}$, the stability condition (pressure zero condition) and the compressibility value of symmetric matter $K_{0}$ at saturation density $\rho_{0}$. The density dependence of the binding energy per nucleon in symmetric matter is shown in Figure 1. One can see that the results are very close to the APR (Akmal-Pandharipande-Ravenhall) predictions in Ref. [3]. Analogously, defining the binding energy per nucleon in asymmetric matter as

$$
\varepsilon_{V}(\lambda)+\varepsilon_{A}(\lambda, \delta) \equiv \frac{Z m_{\mathrm{p}}^{*}(\lambda, \delta)+N m_{\mathrm{n}}^{*}(\lambda, \delta)}{A}-m_{N}=m_{N, s}^{*}(\lambda, \delta)+\delta m_{N, v}^{*}(\lambda, \delta)-m_{N},
$$

where $N$ is the number of neutrons, $Z$ is the number of protons and $A$ is the number of nucleons, one can also reproduce asymmetric matter properties. The results are consistent with the results of successful phenomenological approaches [3, 4] and more discussions can be found in Refs. [2, 6].

At this stage we are ready to make the predictions about the structure changes of the in-medium nucleons. For example, the isoscalar $(I=0)$ and isovector $(I=1)$ electric $(E)$ charge densities have the following forms [6]

$$
\begin{aligned}
& \rho_{E, I=0}(x)=-\frac{2}{\pi} s^{2} F_{x}=\tilde{B}(x) \\
& \rho_{E, I=1}^{*}(x)=\frac{\tilde{\Lambda}(x)}{\Lambda}+2\left(\tilde{\Lambda}_{e}^{*}(x)-\Lambda_{e}^{*} \frac{\tilde{\Lambda}(x)}{\Lambda}\right)
\end{aligned}
$$




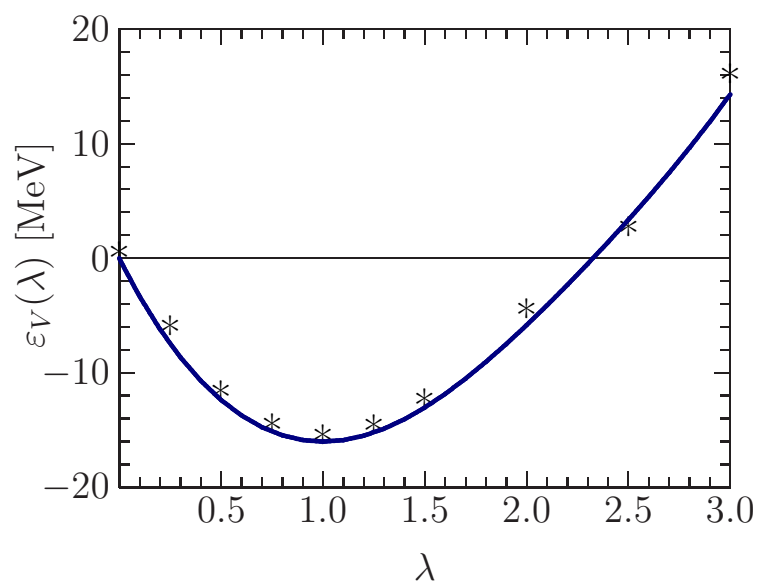

Figure 1: (Color online) The volume energy as a function of normalized density $\lambda=\rho / \rho_{0}$. Solid curve corresponds to the value of compressibility $K_{0}=240 \mathrm{MeV}$. The variational parameters have values $C_{1}=$ $-0.279, C_{2}=0.737$ and $C_{3}=1.782$. Akmal-Pandharipande-Ravenhall predictions [3] are marked by stars.

Here the quantities with a "tilde" are the corresponding integrands, e.g. $\Lambda=\int_{0}^{\infty} \tilde{\Lambda}(x) \mathrm{d} x$. Consequently, the mean-square-electric-charge radii are given as

$$
\begin{aligned}
\langle r\rangle_{E, I=0}^{* 2} & =\left(\frac{f_{1}}{\alpha_{s}}\right)^{2} \frac{1}{e^{2} F_{\pi}^{2}} \int_{0}^{\infty} \tilde{B}(x) x^{2} \mathrm{~d} x \\
\langle r\rangle_{E, I=1}^{* 2} & =\left(\frac{f_{1}}{\alpha_{s}}\right)^{2} \frac{1}{e^{2} F_{\pi}^{2}} \int_{0}^{\infty} \rho_{E, I=1}^{*}(x) x^{2} \mathrm{~d} x .
\end{aligned}
$$

Analogously, the normalized magnetic (M) moment densities have forms [6]

$$
\rho_{M, I=0}(x)=\frac{\tilde{B}(x) x^{2}}{\int_{0}^{\infty} \tilde{B}(x) x^{2} \mathrm{~d} x}, \quad \rho_{M, I=1}(x)=\frac{\tilde{\Lambda}(x)}{\Lambda}
$$

and the mean-square-magnetic-charge radii are given as

$$
\langle r\rangle_{M, I=0,1}^{* 2}=\left(\frac{f_{1}}{\alpha_{s}}\right)^{2} \frac{1}{e^{2} F_{\pi}^{2}} \int_{0}^{\infty} \rho_{M, I=0,1}(x) x^{2} \mathrm{~d} x .
$$

The outcome results are presented in Table 1, where it has been used the simple parametrization $\alpha_{s}=\exp \{-0.65 x\}$. This parametrization is consistent with the parametrizations from the pionic atoms data up to normal nuclear matter densities (for more explanations, see Ref. [6]).

One can see that the sizes of the nucleons are increased in nuclear matter. The changes in isoscalar-root-mean-square radii are large in comparison with the changes in isovector-root-meansquare radii. In neutron matter, only the isovector-electric-charge-radius values change in comparison with the values in symmetric matter. In symmetric matter the changes in isovector-electric and isovector-magnetic radii are same, while in asymmetric matter the changes in isovector-electric radius are more pronounced due to the additional medium effects (see Eq. (4.4)). The results are consistent with the phenomenological indications [1].

In summary, we have discussed very simple five parametric model of asymmetric nuclear matter based on the in-medium modified Skyrme Lagrangian. The reproduced properties of symmetric 


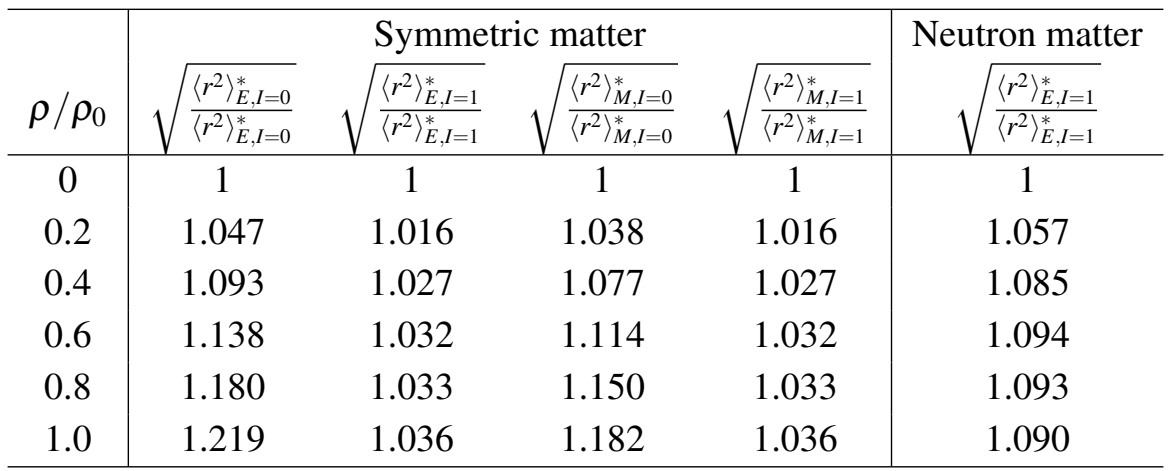

Table 1: The ratio of the in-medium-root-mean-square radii of the nucleons to those in free space as a function of normalized density $\lambda=\rho / \rho_{0}$. The variational parameters have values: $C_{1}=-0.279, C_{2}=0.737$, $C_{3}=1.782, C_{4}=1.541$ and $C_{5}=0.113$. The results in neutron matter are same as in symmetric matter except the isovector-electric-root-mean-square radius.

and asymmetric matter are found to be very reasonable and consistent with other model calculations and the experimental indications (for more discussions, see Refs. [2, 6]). The basic future of the present model is a treatment of the properties of many nucleonic systems and the single nucleon properties in nuclear matter at same footing starting from the same Lagrangian. The model can be applied to the studies of nucleon properties in finite nuclei as well as to the analysis of finite nuclei properties.

\section{Acknowledgments}

This work is supported by the Basic Science Research Program through the National Research Foundation (NRF) of Korea funded by the Korean government (Ministry of Education, Science and Technology), Grant No. 2012-0008469.

\section{References}

[1] J. J. Aubert et al. [European Muon Collaboration], The ratio of the nucleon structure functions $F 2_{n}$ for iron and deuterium, Phys. Lett. B 123 (1983) 275.

[2] U. T. Yakhshiev, In-medium nucleons and nucleonic systems: Infinite nuclear matter, Phys. Rev. C 88 (2013) 034318.

[3] A. Akmal, V. R. Pandharipande and D. G. Ravenhall, The Equation of state of nucleon matter and neutron star structure, Phys. Rev. C 58 (1998) 1804 [nucl-th/9804027].

[4] P. Danielewicz, R. Lacey and W. G. Lynch, Determination of the equation of state of dense matter, Science 298 (2002) 1592.

[5] J.-H. Jung, U. T. Yakhshiev and H.-Ch. Kim, In-medium modified $\pi-\rho-\omega$ mesonic Lagrangian and properties of nuclear matter, Phys. Lett. B 723 (2013) 442 [arXiv:1212.4616 [hep-ph]].

[6] U. T. Yakhshiev, Medium modification of nucleon properties in the Skyrme model revisited, in preparation. 